\title{
Brain activity and connectivity differences in reward value dis- crimination during effort computation in schizophrenia
}

Cite this article as: Clara Pretus, Daniel Bergé, Xavier Guell, Victor Pérez and Óscar Vilarroya, Brain activity and connectivity differences in reward value discrimination during effort computation in schizophrenia, European Archives of Psychiatry and Clinical Neuroscience https://doi.org/10.1007/s00406-020-01145-8

This Author Accepted Manuscript is a PDF file of an unedited peer-reviewed manuscript that has been accepted for publication but has not been copyedited or corrected. The official version of record that is published in the journal is kept up to date and so may therefore differ from this version.

Terms of use and reuse: academic research for non-commercial purposes, see here for full terms. https://www.springer.com/aam-terms- 1 


\title{
Brain activity and connectivity differences in reward value discrimination during effort computation in schizophrenia
}

\author{
Clara Pretus ${ }^{\mathrm{a}, \mathrm{b}^{*}}$, Daniel Bergé $e^{\mathrm{a}, \mathrm{b}, \mathrm{c}, \mathrm{d}^{*}}$, Xavier Guell ${ }^{\mathrm{a}, \mathrm{e}, \mathrm{f}}$, Victor Pérez ${ }^{\mathrm{a}, \mathrm{b}, \mathrm{c}, \mathrm{d}}$, Óscar Vilarroya $\mathrm{a}^{\mathrm{a}, \mathrm{b}}$
}

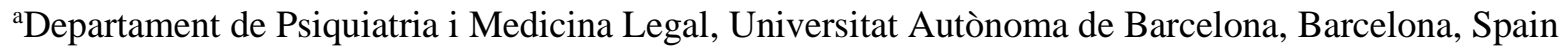

${ }^{\mathrm{b}}$ Hospital del Mar Medical Research Institute (IMIM), Barcelona, Spain.

'Institut de Neuropsiquiatria i Addiccions, Hospital del Mar, Barcelona, Spain

${ }^{\mathrm{d}}$ Centro de Investigación Biomédica en Red de Salud Mental, CIBERSAM G21, Spain

'Department of Brain and Cognitive Sciences and McGovern Institute for Brain Research, Massachusetts Institute of Technology, Cambridge, MA, USA

${ }^{\text {fH}}$ Harvard Medical School and Massachusetts General Hospital, Boston, MA, USA

*shared first authorship

\section{Corresponding author:}

Clara Pretus

clara.pretus@gmail.com

ORCID: 0000-0003-2172-1184

\section{Acknowledgments}

We would like to thank all the volunteers who kindly agreed to participate in this study. This work was supported by the Instituto de Salud Carlos III (ISCiii), Proyectos de Investigación y Salud, grant reference PI18/00053. 


\begin{abstract}
Negative symptoms in the motivational domain are strongly correlated with deficits in social and occupational functioning in schizophrenia. However, the neural substrates underlying these symptoms remain largely unknown.

Twenty-eight adults with schizophrenia and 20 healthy volunteers underwent functional magnetic resonance while completing a lottery game designed to capture reward-related cognitive processes. Each trial demanded an initial investment of effort in form of key presses in order to increase the odds of winning. Brain activity in response to different reward cues (1 euro versus 1 cent) was compared between groups.

Whereas controls invested more effort in improving their chances to win 1euro compared to 1 cent in the lottery game, patients invested similarly high amounts of effort in both reward conditions. The neuroimaging analysis revealed lower neural activity in the bilateral caudate and cingulo-opercular circuits and decreased effective connectivity between reward-associated areas and neural nodes in the frontoparietal and salience network in response to high versus low reward conditions in schizophrenia patients compared to controls. Effective connectivity differences across conditions were associated with amotivation symptoms in patients.

Overall, our data provide evidence of alterations in neural activity in the caudate and cingulo-opercular "task maintenance" circuits and frontoparietal effective connectivity with reward-associated nodes as possible underlying mechanisms of reward value discrimination deficits affecting effort computation in schizophrenia.
\end{abstract}

\title{
Keywords
}

Schizophrenia; Effort-based task; Effective connectivity; Effort computation; Amotivation 


\section{Introduction}

Negative symptoms in schizophrenia include two main components: inexpression and amotivation [1,2]. The latter encompasses asociality, anhedonia and avoltion symptoms, which have been pointed out as strong contributors to social and occupational functioning impairments in schizophrenia [3-5], a disorder with a 4.6/1000 point prevalence [6]. At a cognitive level, amotivation symptoms have been generally associated with impairments in reward processing [7] and, specifically, goal-oriented behavior [3, 8, 9]. Unfortunately, current treatments have a very limited impact on motivational symptoms, hampering improvement in patients' everyday function [10]. Such inefficiency is partly related to the fact that neural substrates underlying goaloriented behavior in schizophrenia remain largely unknown.

Schizophrenia has been associated with impairments in both of the main components of goal-directed behavior: value computation and effort computation [11]. A series of studies support the idea that motivation deficits in schizophrenia may be related to deficiencies in value computation of available options $[9,12,13]$. Conversely, Fervaha et al. [3] also found that, independently from reward valuation, patients compared to controls more frequently chose easy trials that involved lower effort (in form of keypresses) to win smaller amounts of money with a given probability than hard trials (more effort and higher rewards), pointing at impairments in effort computation. Using a similar task, Gold et al. [8] and Barch et al. [14] found that this pattern was insensitive to, respectively, reward certainty and increases in probability of winning or increases in reward value. Moreover, Wolf et al. [15] employed a task where participants chose to engage or not in a given trial that presented an amount of effort (performing a cognitive task a given amount of times) to earn a given amount of money. In this study, maximal effort for a particular reward was lower in schizophrenia patients compared to controls.

However, because each trial offers pre-defined options involving a given amount of effort for a given amount of money, these effort-based tasks do not allow participants to adjust the amount of effort they are willing to invest to directly affect the odds of earning a given reward, an approach that seems more ecological to capture motivation to attain costly rewards in daily life. Thus, we developed an effort-based task that allows participants to select one out of three available effort levels in order to increase their odds of earning a given reward [16]. Since none of the above tasks has been directly tested under the fMRI scanner, we used our effortbased task to shed light into the brain areas that might be involved in effort computation alterations in schizophrenia. Importantly, because negative symptoms extend to other disorders, such as Parkinson's disease, Alzheimer's disease, multiple sclerosis or cerebrovascular lesions [17], advancements in the understanding of negative symptoms in schizophrenia could have trans-diagnostic value.

Neurally, we expected our effort-based task to elicit activity in different brain regions involved in goal directed behavior. As part of goal-directed behavior, value computation has been associated with orbitofrontal activity [18], while effort computation has been related to basal ganglia and anterior cingulate cortex activity [19, 20], which has been found to signal the interaction between expected rewards and effort costs [21]. In addition, anterior cingulate cortex activity, together with the supplementary motor activity, has been found to encode differences in reward and amount of effort between different options [22]. 
In schizophrenia, most of the neuroimaging studies targeting motivation focus on the ventral striatum using region of interest analysis techniques. For instance, Morris et al. [23] found exaggerated right ventro-striatal response to expected rewards and blunted left ventro-striatal response to unexpected rewards in schizophrenia patients compared to controls. Moreover, several studies describe attenuated ventro-striatial activity in response to reward-indicating cues in schizophrenia, which correlated, respectively, with positive symptoms [24] and negative symptoms [15, 25]. However, no differences in ventral striatal volume were found in patients compared to controls [26]. Aside from the ventral striatum, reward-predicting cues were associated with blunted basal ganglia activation in schizophrenia, with amotivation symptoms predicting reduced sustained dorsolateral prefrontal activity during a reward task [27]. Patients also showed reduced striatal and anterior cingulate activity during reward approach initiation, which correlated with amotivation symptoms using a reinforcement learning task [28]. In line with the trans-diagnostic value of negative symptom research [29], Segarra et al. [30] found reduced orbitofrontal and striatal activity in both depression and schizophrenia patients in response to unexpected rewards, with stronger alterations in medial prefrontal activity in the latter group.

Finally, studies focusing on connectivity and reward processing in schizophrenia, though scarce and based on small samples, suggest a dysconnectivity pattern between ventral regions of the reward system and the prefrontal cortex [31, 32]. However, whether and how functional connectivity is affected during effort computation in schizophrenia and how it relates to negative symptoms remains poorly understood. Elucidating these questions may contribute to a better understanding of motivation deficits in schizophrenia and facilitate the design of future therapeutic strategies.

\section{Aims of the Study}

The aim of the present study was to identify the neural substrates underlying deficits in goal-directed behavior in schizophrenia, circumventing the effect of cognitive effort. For this purpose, brain activity and connectivity was evaluated by means of functional magnetic resonance in sample of schizophrenia patients compared to healthy volunteers as they completed a task specifically designed to capture participants' willingness to invest varying amounts of effort to directly affect the odds of earning either high or low rewards with minimal amounts cognitive effort.

\section{Materials and Methods}

\section{Participants}

Twenty-eight adults with schizophrenia (10 women) and 20 healthy subjects (10 women) were recruited for the study (see demographics in Table 1). Both groups were homogenous for gender. The schizophrenia patients 
were recruited from the outpatient services of psychiatry at the Hospital del Mar in Barcelona [33]. All of them met the DSM-IV criteria [34] for schizophrenia.

Symptom severity was assessed by means of the Brief Negative Symptom Scale (BNSS) [35], global functioning was evaluated using the Global Assessment of Functioning (GAF) [36] and diagnostic was confirmed with the SCID interview for diagnosis of schizophrenia and affective spectrum disorders [37]. All patients were undergoing antipsychotic treatment, most of them with atypical antipsychotics (12 with aripiprazole, 4 with clozapine, 3 with olanzapine, 3 with paliperidone, 1 with risperidone and 1 with ziprasidone) and only three with typical antipsychotics (1 with fluphenazine and 2 with zuclopenthixol). Data regarding antipsychotic treatment were recorded and converted to chlorpromazine milliequivalents.

Exclusion criteria included comorbidity with other psychiatric diseases or personality disorders, assessed by the Mini-International Neuropsychiatric Interview (M.I.N.I) [38], inability to fully comprehend the task procedure and goals, and ages above 50 years old. Participants with substance abuse disorder or in an acute relapse of their psychotic disorder (above 3-point score in any PANSS positive item [39]) were also excluded. Cigarette consumption was not controlled for.

\section{fMRI paradigm}

The rapid event related fMRI paradigm included randomized trials and jittered inter-trial intervals (ITI) and was designed using Matlab with Psychtoolbox extensions [40]. Participants were trained to complete the task and practiced it thoroughly for about 5 minutes just before going into the fMRI scanner. Participants did not show any signs of tiredness after the training, which was apparent in the high levels of effort selected during the fMRI task in both patients and controls.

The paradigm was divided in two 14-min long runs, each including a hundred 14-second randomly spaced trials. Each trial consisted of an adapted lottery game with two conditions: playing either for 1cent or for leuro. At the beginning of the trial, participants were presented the amount of money for which they were going to play (either 1 cent or 1euro). Next, they had to choose, among 3 options, the number of times they were willing to press the key (amount of effort) for the money at stake ( 1 cent or 1 euro). The lowest effort option (Effort A) required pressing the key 6 times, the intermediate option (Effort B) 16 times and the highest effort option (Effort C) 45 times. Such sequential increase was calculated as a function of the natural logarithm and based on previous behavioral testing [41]. In order to diminish automatic responses, two more variations of Effort $\mathrm{A}, \mathrm{B}$ and $\mathrm{C}$ were included, comprising a 5\% increase (6,17 and 47 key presses) and a 5\% decrease $(6,15$ and 43 key presses) with respect to the original values, which did not affect performance. After choosing the Effort option, participants were required to press the key accordingly (effort investment). If the Effort was not completed in due time (within $8000 \mathrm{~ms}$ ), the trial was counted as a missing. Time constraints were designed so that participants would have enough time to complete all required key presses. Next, the lottery game started. The odds of winning the game reflected the Effort option selected at the start of the trial (1/4 for Effort A, 2/4 
for Effort B and 3/4 for Effort C). At the end of the trial, the result of the lottery game and the participant's accumulated gain was presented (see detailed trial sequence in Figure 1). The total gain, which ranged between 1 and 42.43 euros, was reimbursed at the end of the game. If participants accumulated a gain below 25 euros, they were reimbursed 25 euros. Payment conditions were the same for both patients and controls.

\section{Analysis of performance data}

In order to assess motivational differences between schizophrenia patients and control participants, selected effort level in each trial was taken as a proxy of motivation and evaluated in relationship to the expected outcome of the game (1euro vs. 1 cent). For this purpose, a two-way repeated measures ANOVA was conducted on selected effort level with group membership as a between-subjects factor, expected reward value (1euro vs. 1 cent) as a within-subjects factor, and age and sex as covariates.

For each individual, frequencies of optimal choices (maximal effort for 1euro and minimal effort for 1cent), intermediate choices (intermediate effort for both 1euro and 1cent) and sub-optimal choices (minimal effort for 1euro and maximal effort for 1cent) were calculated and differences between groups were compared by means of independent sample t-tests. Moreover, total effort (in number of total keypresses), total gain (in euros) and efficiency (total gain/total effort) were compared between groups.

Furthermore, in order to rule out possible confounding effects of the different variants of the proposed amount of key presses (6-15-43 vs. 6-16-45 vs. 6-17-47) on effort level selection, a further two-way repeated measures ANOVAs was conducted with effort level as the dependent variable, group membership as between-subjects factor and key press variant as within-subjects factor.

\section{fMRI image acquisition and analysis}

Images were acquired in a Siemens 3T scanner. T1-weighted images were obtained using a FSPGR sequence (TR: $11.6 \mathrm{~ms}$, TE: $4.8 \mathrm{~ms}$, FA: 12, matrix size: 280 x 280, 150 slices, slice thickness: $1.00 \mathrm{~mm}$ ). An EPI-T2* sequence allowed obtaining the functional volumes, each comprising forty $3.4 \mathrm{~mm}$ thick slices (TR $2500 \mathrm{~ms}$, TE: 27 ms, FA: 90, matrix size: 64 x 64, 40 slices, slice thickness: $3.4 \mathrm{~mm}$ ). Head motion was tracked by means of center of mass measurements during image acquisition, allowing us to repeat sequences if necessary.

Functional MRI data were analyzed with the software package SPM12 (Welcome Department of Imaging Neuroscience, London, United Kingdom) on Matlab R2017a (MathWorks Inc., Natick, Massachusetts). Functional images were realigned to the mean image in order to correct for motion-related artifacts, coregistered to the structural image, normalized to MNI standard space and spatially smoothed with an $8 \mathrm{~mm}$ fullwidth-at-half-maximum Gaussian kernel. Groups did not differ in mean head movement (patients: $\mathrm{M}=0.20$, $\mathrm{SD}=0.14$; controls: $\mathrm{M}=0.22, \mathrm{SD}=1.46$, mean diff $=-0.018, \mathrm{t}(46)=-.433, \mathrm{p}=.667$ ) nor in maximal head 
movement (patients: $\mathrm{M}=1.63, \mathrm{SD}=1.27$; controls: $\mathrm{M}=1.77, \mathrm{SD}=1.36$, mean diff $=-0.134, \mathrm{t}(46)=-.352, \mathrm{p}$ $=.727)$.

The GLM design included (1) two different regressors for leuro and 1 cent reward presentation events (750ms), (2) two regressors for effort level selection events for 1 euro and for 1 cent (2500ms), each parametrically modulated by selected effort level $(1=\mathrm{A}, 2=\mathrm{B}$ or $3=\mathrm{C})$, (3) one regressor for effort execution events (up to $8000 \mathrm{~ms}$ ) parametrically modulated by number of keypresses in each trial, (4) one regressor for the lottery event (900-1200ms), and (5) one regressor for the result event (You win/You lose, 750ms) parametrically modulated by a vector including the values -100 (You lose/1euro), -1 (You lose/1cent), 0 (missing trial), +1 (You win/1cent) and +100 (You win/1 euro), together with (6) six movement parameters.

In order to assess possible motivational differences between groups at a neural level, individual level brain activity contrasts, including a) the 1euro vs. 1cent reward presentation events, b) effort selection events for 1 euro vs. 1 cent, and c) the result as a parametric modulator, were submitted to a second-level two-sample t-test with group membership as a between-subjects factor and age as a covariate of no interest. Because antipsychotics block D2 receptors in ventral striatum [42], medication dose in chlorpromazine milliequivalents was also added as a co-variate of no interest. Moreover, in the sample of patients, three second-level regressions were conducted with, respectively, the total BNSS score, the expression and motivation dimension scores as predictors of brain activity in each of the contrasts of interest (a-c), controlling for medication and age. These analyses were used to evaluate the possible predictive value of these symptom scales on measurable neural activity in schizophrenia patients. All second level analyses were corrected for multiple comparisons by means of cluster-level Family-wise error correction, using a peak-level threshold of $\mathrm{p}<0.001$.

\section{Effective connectivity analysis}

The effective connectivity analysis was conducted by means of the connectivity toolbox CONN 18.b (Whitfield-Gabrieli and Nieto-Castanon, 2012) on SPM12. Using this tool, functional images were denoised by regressing out five components derived from white matter, five components from CSF, six motion parameters, noise components related to session and condition effects and any volume with an FD value higher than 0.9 (outliers). To compare the interplay between brain regions associated with reward processing and brain networks related to attention and task control in schizophrenia patients and controls, a series of sixteen ROIs were defined. First, a functional ROI for "reward" was extracted from Neurosynth [43], a platform that calculates probability maps reflecting the likelihood that a term such as 'reward' is used in a study based on the activation in a given voxel by evaluating 14371 different studies. We thresholded the probability map associated with "reward" at $\mathrm{p}<0.05$ and picked the ventral striatum cluster using xjview (https://www.alivelearn.net/xjview) on SPM12. The resulting ROI included the bilateral nucleus accumbens and the immediately surrounding regions (see Figure 4B). Next, we selected potential candidates to modulate reward-associated activation during goal-directed behavior including the standard ROIs in the dorsal attention, the salience and the frontoparietal network provided by the CONN toolbox. These standard ROIs include the anterior cingulate cortex, the bilateral anterior insula, the bilateral rostral prefrontal cortex and the bilateral 
supramarginal gyrus for the salience network; the bilateral frontal eye fields and the bilateral inferior parietal sulcus for the dorsal attention network; and the bilateral lateral prefrontal cortex and the bilateral posterior parietal cortex for the frontoparietal network (see Figure 3B). For each participant, a generalized PsychoPhysiological Interaction (gPPI) analysis [44] was conducted. This type of analysis allows to quantify how brain regions interact in a task-dependent manner by modeling the task effects, the ROIs timeseries and an interaction term (PPI term) between both parts by means of a multiple regression model. Here, the effects of the task conditions ( 1 euro compared to 1 cent) during reward presentation and effort selection on effective connectivity between the evaluated ROIs were then computed and compared. Group-level gPPI results were corrected for multiple comparisons at seed level ( $\mathrm{p}<0.05$ FDR).

\section{Results}

\section{Behavioral results}

Reward condition ( 1 euro vs. 1 cent) had a significant effect on effort level selection across groups (Wilk's Lambda $=0.746, \mathrm{~F}(44,1)=15, \mathrm{p}<0.001)$ with higher reward value associated with higher effort level (1 euro: mean effort level $(\mathrm{sd})=2.73(0.37), 1$ cent: mean effort level $(\mathrm{sd})=2.08(0.62))$. In turn, the interaction between reward condition and group membership had a significant effect on effort level selection (Wilk's Lambda = 0.887, $\mathrm{F}(44,1)=5.62, \mathrm{p}<0.022$ ) in a two-way repeated measures ANOVA controlling for age and sex (see Figure 2A), with patients exhibiting lower differences in effort level for 1 euro compared to 1 cent (mean(sd) leuro $_{\text {( }}$ $=2.67(0.39)$ vs. mean $\left.(\mathrm{sd})_{\text {lent }}=2.28(0.48)\right)$ compared to controls (mean $(\mathrm{sd})_{\text {leuro }}=2.81(0.32) \mathrm{vs}$. mean $(\mathrm{sd})_{\text {lcent }}=$ $1.82(0.7))$. No significant between-group differences were detected in mean effort level across conditions.

Indeed, percentage of optimal choices, including maximal effort level for 1 euro and minimal effort level for 1 cent, was lower in patients $($ mean $(\mathrm{sd})=44 \%(0.19))$ than controls $($ mean $(\mathrm{sd})=62 \%(0.22), \mathrm{t}(46)=2.882, \mathrm{p}$ $=0.006$, see Figure $2 \mathrm{~B}$ ). Between-group differences in suboptimal (maximal effort for 1 cent and minimal effort for 1 euro) and intermediate choices (intermediate effort for either 1 cent or 1 euro) did not survive Bonferroni correction. Patients made less optimal choices primarily because they chose higher effort levels for 1 cent $($ mean $(\mathrm{sd})=2.28(0.48))$ than controls $($ mean $(\mathrm{sd})=1.81(0.7), \mathrm{t}(31,26)=-2.58, \mathrm{p}=0.015)$, whereas no between-group differences were detected in mean effort level for 1 euro.

Efficiency, calculated as "total gain/total effort" was lower in patients (mean $(\mathrm{sd})=0.009(0.004))$, than in controls (mean $(\mathrm{sd})=0.012(.005), \mathrm{t}(46)=2.84, \mathrm{p}=0.007)$, and it was negatively associated with suboptimal choices (minimal effort for 1euro and maximal effort for 1cent), in both samples (controls: $\mathrm{r}=-0,682, \mathrm{p}=$ 0.001, patients: $\mathrm{r}=-0.597, \mathrm{p}=0.001$ ).

Total gain in euros was different between groups, with patients earning less (mean(sd) $=24.90(12.87)$ ) than controls $(\mathrm{mean}(\mathrm{sd})=31.85(8.39), \mathrm{t}(45.69)=2.26, \mathrm{p}=0.028)$. In patients, total gain positively correlated with 
total effort or number of keypresses per participant $(\mathrm{r}=0.628, \mathrm{p}<0.001$, see Figure $2 \mathrm{C})$, whereas it correlated with optimal choices in controls $(r=.543, p=0.013$, see Figure 2D). Total effort did not differ betweengroups, although it was negatively associated with inexpressiveness $(r=-0.483, p=0.009)$ and total BNSS $(r$ $=-0.425, \mathrm{p}=0.024)$ in patients and positively correlated with suboptimal choices $(\mathrm{r}=0.758, \mathrm{p}<0.001)$ in controls.

When looking at the effect of potential confounds, no association was found between medication (in chlorpromazine milliequivalents) and any of the task outcomes. However, medication was correlated with total BNSS $(r=.450, p=.016)$, expression $(r=.433, p=0.021)$, and amotivation as a trend $(r=.369, p=.053)$. Furthermore, the repeated measures ANOVA adjusted for group membership, age and sex, did not identify any effects related to the key press permutations (6-15-43 vs. 6-16-45 vs. 6-17-47). The expected probabilities of winning the lottery game (1/4 for effort level 1, 2/4 for effort level 2 and 3/4 for effort level 3) were confirmed by a post-hoc analysis of the participants' outcomes.

\section{Neuroimaging results}

In schizophrenia patients compared to controls, the neuroimaging whole-brain analysis revealed lower neural activity differences between reward conditions ( 1 euro versus 1 cent) during reward presentation events in the right caudate extending into the right superior anterior cingulate cortex and the right inferior frontal gyrus $(\mathrm{k}$ $=1003, \mathrm{~T}=4.79, \mathrm{p}<0.05 \mathrm{FWEc}$, single voxel $\mathrm{p}<0.001$ ), as well as a trend towards significance in the left caudate extending into the left insula and left superior anterior cingulate cortex $(\mathrm{k}=309, \mathrm{~T}=5.44, \mathrm{p}=0.06$ FWEc, single voxel $\mathrm{p}<0.001$; see Table 2 and Figure 3A).

Similarly, schizophrenia patients compared to controls also showed lower neural activity differences between reward conditions ( 1 euro versus 1 cent) during effort selection events in the left caudate extending into the left insula $(\mathrm{k}=618, \mathrm{~T}=5.00, \mathrm{p}<0.05 \mathrm{FWEc}$, single voxel $\mathrm{p}<0.001)$ and the right caudate extending into the right inferior frontal gyrus (pars triangularis) and the right middle frontal gyrus $(\mathrm{k}=1595, \mathrm{~T}=4.67, \mathrm{p}<0.05$ FWEc, single voxel $\mathrm{p}<0.001$; see Table 2 and Figure 3B). Parameter estimates extraction revealed that such between-group differences were mostly driven by reduced neural recruitment for higher rewards ( 1 euro) in schizophrenia patients in both left and right lateralized clusters of activity (see Figure 3B). A post-hoc analysis using a "reward" functional ROI extracted from Neurosynth involving the bilateral nucleus accumbens and its circumventing areas did not show between-groups differences, although it yielded significant activity during effort selection across groups $(\mathrm{k}=137, \mathrm{~T}>3.29$, single-voxel $\mathrm{p}<0.001$, small volume correction). Analysis of the lottery and lottery result events, as well as the effort level parametric modulator, did not reveal any significant effects of the interaction between group membership and reward condition ( 1 euro vs. 1 cent). Symptom severity scores and medication in the patients' sample, as well as age across groups, did not predict any significant activity. 
The gPPI analysis revealed decreased effective connectivity between the "reward" functional ROI extracted from Neurosynth and both frontoparietal network regions (right lateral prefrontal cortex and bilateral posterior parietal cortex) and salience network regions (right supramarginal gyrus) in patients compared to controls during effort selection in the 1 euro compared to the 1 cent condition $(\mathrm{p}<0.05$ FDR seed-level corrected, see Figure 4A). In the same contrast, patients also exhibited decreased effective connectivity between nodes in the dorsal attention network (left inferior parietal sulcus) and in the frontoparietal network (right lateral prefrontal cortex) compared to controls ( $\mathrm{p}<0.05$ FDR seed-level corrected, see Figure 4A). Connectivity results between conditions during the reward presentation event did not survive multiple comparisons correction. gPPI parameter estimates extraction showed reduced reward value discrimination in patients compared to controls in terms of connectivity between the "reward" ROI and the right lateral prefrontal cortex during effort selection. Particularly, controls exhibited a markedly different effective connectivity pattern between these two regions as a function of reward value, whilst patients showed similar patterns across reward conditions (see parameter estimates in Figure 4A). Moreover, amotivation symptoms were associated with decreased effective connectivity change between the "reward" ROI and the right lateral prefrontal cortex in response to high versus low reward condition $(r=-0.567, p=0.002)$ during effort selection after Bonferroni multiple comparisons correction.

\section{Discussion}

In this $2 \times 2$ mixed factorial design study, we evaluated reward value representation and effort computation in a sample of schizophrenia patients compared to controls using a lottery game. We found that controls were more discriminative towards reward value by selecting higher effort levels for the high reward condition (1euro) and lower effort levels for the low reward condition (1cent), while patients were less discriminative, especially since they selected, on average, much higher effort levels for the minimal reward than controls. At a neural level, this phenomenon was associated with reduced activity change in response to different reward conditions in schizophrenia patients compared to controls, both in terms of neural activity and effective connectivity. Particularly, schizophrenia patients showed lower neural activity change in the bilateral caudate extending into the right inferior frontal gyrus and the left insula across reward conditions compared to controls, both during reward presentation and effort selection events. In addition, patients (versus controls) showed lower effective connectivity change across reward conditions between "reward" associated regions, including the bilateral nucleus accumbens and its circumventing areas, and frontoparietal network regions such as the right lateral prefrontal cortex during effort selection events.

Several studies, including a study by our group using an adaptation of the same effort-based task [16], report lower disposition to invest effort in schizophrenia patients compared to controls [15], even when reward value representation is preserved [3]. However, the present study did not reveal group related differences in total effort, that is, disposition to invest effort was similar in both groups. Such discrepancies could be related to the task being adapted for fMRI acquisition. The fMRI task involved fixed effort execution periods ( 8 seconds) so that all patients were able to complete the task while their brains were being scanned without abandoning the 
task. Conversely, effort execution periods increased exponentially with effort level in the task version employed in our previous behavioral study [16], and thus, task completion required substantially more effort. In addition, our present findings support the notion that schizophrenia patients are less discriminative when it comes to investing effort to attain rewards with different values. Specifically, percentage of optimal choices, including the selection of maximal effort levels for 1 euro and minimal effort levels for 1 cent, was higher in control participants than patients, who barely made a distinction between reward values in terms of selected effort. Group differences in suboptimal choices (minimal effort for 1 euro and maximal effort for 1 cent) did not survive multiple comparisons correction, suggesting that patients were more often investing too much effort for 1 cent than too little effort for 1 euro. Overall, our behavioral results indicate that discriminative allocation of effort in response to different reward values may be impaired in schizophrenia patients, which could be due to deficiencies in reward value discrimination in the context of effort computation, as suggested by the neuroimaging findings.

When evaluating brain activity, both reward presentation and effort selection events were associated with reduced differences in neural activity across reward conditions in patients compared to controls, even after controlling for selected effort level in each trial (A, B or C). Such between-group neural activity differences were found bilaterally in the caudate and the anterior cingulate cortex extending into the left insula and the right inferior and middle frontal gyrus, respectively. Moreover, these results were driven by decreased neural activity in these clusters in response to the high reward condition (1euro) in patients, who otherwise showed similar neural activity levels in response to the low reward condition (1 cent) relative to controls. These findings suggest a deficient recruitment of the caudate in front of high rewards in schizophrenia patients, which could be affecting planning of goal-directed action in this sample, in line with the view of the caudate nucleus as a main neural node subserving goal directed behavior [45-47]. Moreover, our results are aligned with studies pointing at the role of the basal ganglia in effort computation [20].

Aside from the caudate, the involvement of other regions comprising the insula and the anterior cingulate cortex leads to complementary interpretations. On one hand, the observed neural activity in the anterior cingulate cortex is in line with studies linking anterior cingulate activity with effort-based cost-benefit computation. For instance, anterior cingulate activity has been associated with the interaction between expected rewards and effort costs [21] and with the difference in reward and effort between different options in conjunction with the supplementary motor area in a task incorporating physical effort and monetary rewards [22]. On the other hand, the involvement of both insular regions and the anterior cingulate cortex bilaterally points to a possible role of the cingulo-opercular task control network [48], a system thought to modulate "task maintenance" processes, the activity of which is sustained throughout the completion of the task and increases in tasks that require higher "tonic alertness" [49]. The bilateral activation of cingulo-opercular regions in our data is in line with recent accounts pointing at a bilateral distribution of the cingulo-opercular cortex [49]. The involvement of this network could explain that between-group differences in cingulo-opercular regions were maintained throughout reward presentation and effort selection events. In this respect, sustained neural activity differences in task maintenance nodes could conform a common neural substrate affecting both reward 
presentation and effort selection in schizophrenia, even if each event may depend on different neural mechanisms. Similarly to the caudate, parameter estimates reveal distinctively high neural activity in cinguloopercular regions during 1-euro rewards in controls, both compared to 1 cent rewards in controls and compared to schizophrenia patients across reward conditions. Thus, our results support the notion that, in controls, "task maintenance" mechanisms may be distinctively involved in the high reward condition, which may respond to increased tonic alertness throughout the completion of the task under these circumstances. Conversely, schizophrenia patients exhibited lower activations in this network across reward conditions, which could be interfering with optimal task completion. For instance, schizophrenia patients generally adopted a more rigid approach to the task by choosing maximal effort levels indistinctively for 1 euro and 1 cent more often, as shown by the behavioral results. Such approach may function as a strategy aimed to compensate deficits in task maintenance mechanisms.

Of note, even though the functional reward-associated ROI (including the bilateral nucleus accumbens) did show task-related activation during effort selection across groups, we did not find any significant differences in ROI activation between groups. Such lack of between-group differences contrasts with other studies making use of ROIs [23-25]. Nonetheless, the employed functional reward-associated ROI did yield significant results in the effective connectivity analysis. Because we conducted a generalized psycho-physiological interaction analysis, the results reveal task-dependent changes in effective connectivity between ROIs during our effortbased reward task. Particularly, we found decreased differences in effective connectivity between rewardassociated regions in and around the bilateral nucleus accumbens (ventral striatum) and the right lateral prefrontal cortex in schizophrenia patients compared to controls in response to 1 euro versus 1 cent during effort selection. Controls distinctively adjusted fronto-striatal connectivity in response to reward value, exhibiting stronger connectivity for higher reward values than lower reward values, whilst schizophrenia patients showed stable connectivity estimates across reward conditions during effort selection. In addition, amotivation symptoms in the experimental group correlated with reduced connectivity change between reward-associated regions and the right lateral prefrontal cortex across reward conditions during effort selection, corroborating these results. These results suggest that schizophrenia patients and, in particular, patients with higher amotivation symptoms are also less prone to flexibly adjust functional fronto-striatal connectivity in response to distinct reward values during effort selection. These findings support the notion that patients used a more rigid approach during effort computation, exhibiting non-distinctive neural activity and connectivity profiles and investing similar effort levels across reward conditions. In addition, such frontostriatal connectivity pattern seems to be driven by amotivation symptoms rather than expression symptoms in schizophrenia patients. Other studies suggest abnormal fronto-striatal connectivity in schizophrenia patients compared to controls, including reduced connectivity between the striatum and right medial and lateral aspects of the prefrontal cortex during resting-state [50]. Social amotivation was also found to be a negative predictor of functional connectivity between the substantia nigra/ventral tegmental area and both the striatum and medial and lateral prefrontal cortex [32]. 
Moreover, we found similar between-group connectivity patterns between the reward-associated ROI and other nodes of the frontoparietal network (bilateral posterior parietal cortex) and the salience network (right supramarginal gryus), as well as between the dorsal attentional network (left inferior parietal sulcus) and the frontoparietal network (right lateral prefrontal cortex) during effort selection. These findings reinforce the view that schizophrenia patients were having difficulties adjusting effective connectivity between relevant attentional and task control networks in order to match presented reward values.

The present study is bound to some limitations. The first limitation is that, even though between-group differences were observed in both the behavioral and the univariate neural analyses, amotivation scores did not predict differences between reward conditions in these analyses. Instead, amotivation was associated with fronto-striatal connectivity deficits in response to different reward values during effort computation, in line with the notion of schizophrenia as a brain connectivity disorder [51-54]. This suggests that, when looking at amotivation symptoms specifically, our effort-based reward task is better suited to capture effective connectivity effects rather than behavioral and univariate neural effects of this symptom scale. Thus, the differential ability of our task to capture amotivation-related effects on brain connectivity compared to other outcomes should be taken into account in future research targeting amotivation effects on reward processing.

The second limitation is that the size of the group samples was not equal between groups (patients: $\mathrm{N}=28$ and controls: $\mathrm{N}=20$ ). Therefore, the neural activity estimates of the control group could be less accurate than those of the patients' group, ultimately affecting the comparisons between groups. This should be taken into account when evaluating our results. Another limitation is that the two samples presented slight differences in age (see Table 1). We addressed this confound by introducing age as a second-level co-variate in the behavioral and neuroimaging analyses comparing both samples. Finally, it is worth noting that medication dose correlated with total BNSS, expression and with amotivation as a trend. This suggests that negative symptoms in the present study could be at least partly secondary negative symptoms due to medication $[55,56]$. Specifically, antipsychotic medication is thought to affect negative symptoms through parkinsonism, especially in the expression domain, though little or no effect has been reported on motivation [57]. This is consistent with the relationship we identified between medication and the BNSS scores. In line with our previous study [16], medication was not associated with any of the task outcomes and antipsychotic dose was added as a co-variate of no interest in the neuroimaging analysis, making it unlikely that our neuroimaging results may have been affected by medication."

In sum, our results indicate that schizophrenia patients chose to invest more similar amounts of effort during across different reward values, which may be related to impaired reward value discrimination at a neural level. Accordingly, patients exhibited reduced neural activity change across reward conditions during reward presentation and effort selection, both in the bilateral caudate and in cingulo-opercular circuits, suggesting deficiencies in task maintenance and planning for goal-directed action during the game. Effective connectivity results reinforce the notion of reward value insensitivity during effort selection in this sample, revealing abnormally similar levels of effective connectivity between reward-associated areas and frontoparietal and 
salience networks across reward conditions in schizophrenia. Overall, we provide evidence of the neural activity and connectivity deficits that may partly underlie reward value discrimination deficits in schizophrenia, affecting investment of effort during goal-directed behavior.

\section{Ethical standards}

The study was approved by the Hospital del Mar Medical Research Institute Ethical Committee and was carried out in accordance with the Declaration of Helsinki. After a full explanation of the study, written informed consent was obtained from all participants before taking part in the study.

\section{Conflict of interest}

The authors declare that they have no conflict of interest. 


\section{References}

1. Blanchard JJ, Cohen AS (2006) The structure of negative symptoms within schizophrenia: Implications for assessment. In: Schizophrenia Bulletin. pp 238-245

2. Kirkpatrick B, Fenton WS, Carpenter WT, Marder SR (2006) The NIMH-MATRICS Consensus Statement on Negative Symptoms. Schizophr Bull 32:214-219. https://doi.org/10.1093/schbul/sbj053

3. Fervaha G, Graff-Guerrero A, Zakzanis KK, et al (2013) Incentive motivation deficits in schizophrenia reflect effort computation impairments during cost-benefit decision-making. $\mathrm{J}$ Psychiatr Res 47:1590-1596. https://doi.org/10.1016/j.jpsychires.2013.08.003

4. Foussias G, Remington G (2010) Negative Symptoms in Schizophrenia: Avolition and Occam's Razor. Schizophr Bull 36:359-369. https://doi.org/10.1093/schbul/sbn094

5. Marwaha S, Johnson S (2004) Schizophrenia and employment. Soc Psychiatry Psychiatr Epidemiol 39:337-349. https://doi.org/10.1007/s00127-004-0762-4

6. Saha S, Chant D, Welham J, McGrath J (2005) A Systematic Review of the Prevalence of Schizophrenia. PLoS Med 2:e141. https://doi.org/10.1371/journal.pmed.0020141

7. Strauss GP, Waltz JA, Gold JM (2014) A Review of Reward Processing and Motivational Impairment in Schizophrenia. Schizophr Bull 40:S107-S116. https://doi.org/10.1093/schbul/sbt197

8. Gold JM, Strauss GP, Waltz JA, et al (2013) Negative symptoms of schizophrenia are associated with abnormal effort-cost computations. Biol Psychiatry 74:130-6. https://doi.org/10.1016/j.biopsych.2012.12.022

9. Gold JM, Waltz JA, Prentice KJ, et al (2008) Reward Processing in Schizophrenia: A Deficit in the Representation of Value. Schizophr Bull 34:835-847. https://doi.org/10.1093/schbul/sbn068

10. Jones C, Hacker D, Cormac I, et al (2012) Cognitive behavioural therapy versus other psychosocial treatments for schizophrenia. Cochrane Database Syst Rev CD008712. https://doi.org/10.1002/14651858.CD008712.pub2

11. Kring AM, Barch DM (2014) The motivation and pleasure dimension of negative symptoms: Neural substrates and behavioral outputs. Eur Neuropsychopharmacol 24:725-736. https://doi.org/10.1016/j.euroneuro.2013.06.007

12. Gold JM, Waltz JA, Matveeva TM, et al (2012) Negative symptoms and the failure to represent the expected reward value of actions: Behavioral and computational modeling evidence. Arch Gen Psychiatry 69:129-138. https://doi.org/10.1001/archgenpsychiatry.2011.1269

13. Heerey EA, Bell-Warren KR, Gold JM (2008) Decision-Making Impairments in the Context of Intact Reward Sensitivity in Schizophrenia. Biol Psychiatry 64:62-69. https://doi.org/10.1016/j.biopsych.2008.02.015

14. Barch DM, Treadway MT, Schoen N (2014) Effort, anhedonia, and function in schizophrenia: Reduced effort allocation predicts amotivation and functional impairment. J Abnorm Psychol 123:387-397. https://doi.org/10.1037/a0036299

15. Wolf DH, Satterthwaite TD, Kantrowitz JJ, et al (2014) Amotivation in Schizophrenia: Integrated Assessment With Behavioral, Clinical, and Imaging Measures. Schizophr Bull 40:1328-1337. 
https://doi.org/10.1093/schbul/sbu026

16. Bergé D, Pretus C, Guell X, et al (2018) Reduced willingness to invest effort in schizophrenia with high negative symptoms regardless of reward stimulus presentation and reward value. Compr Psychiatry 87:. https://doi.org/10.1016/j.comppsych.2018.10.010

17. Brown RG, Pluck G (2000) Negative symptoms: The "pathology" of motivation and goal-directed behaviour. Trends Neurosci. 23:412-417

18. Rudebeck PH, Murray EA (2011) Dissociable Effects of Subtotal Lesions within the Macaque Orbital Prefrontal Cortex on Reward-Guided Behavior. J Neurosci 31:10569-10578. https://doi.org/10.1523/JNEUROSCI.0091-11.2011

19. Botvinick MM, Huffstetler S, McGuire JT (2009) Effort discounting in human nucleus accumbens. Cogn Affect Behav Neurosci 9:16-27. https://doi.org/10.3758/CABN.9.1.16

20. Kurniawan IT, Seymour B, Talmi D, et al (2010) Choosing to make an effort: the role of striatum in signaling physical effort of a chosen action. J Neurophysiol 104:313-21. https://doi.org/10.1152/jn.00027.2010

21. Croxson PL, Walton ME, Reilly JXO, et al (2009) Effort-Based Cost - Benefit Valuation and the Human Brain. 29:4531-4541. https://doi.org/10.1523/JNEUROSCI.4515-08.2009

22. Klein-Flugge MC, Kennerley SW, Friston K, Bestmann S (2016) Neural Signatures of Value Comparison in Human Cingulate Cortex during Decisions Requiring an Effort-Reward Trade-off. J Neurosci 36:10002-10015. https://doi.org/10.1523/JNEUROSCI.0292-16.2016

23. Morris RW, Vercammen A, Lenroot R, et al (2012) Disambiguating ventral striatum fMRI-related bold signal during reward prediction in schizophrenia. Mol Psychiatry 17:280-289. https://doi.org/10.1038/mp.2011.75

24. Nielsen MØ, Rostrup E, Wulff S, et al (2012) Alterations of the Brain Reward System in Antipsychotic Naïve Schizophrenia Patients. Biol Psychiatry 71:898-905. https://doi.org/10.1016/J.BIOPSYCH.2012.02.007

25. Juckel G, Schlagenhauf F, Koslowski M, et al (2006) Dysfunction of ventral striatal reward prediction in schizophrenia. Neuroimage 29:409-416. https://doi.org/10.1016/j.neuroimage.2005.07.051

26. Burrer A, Caravaggio F, Manoliu A, et al (2020) Apathy is not associated with reduced ventral striatal volume in patients with schizophrenia. medRxiv 2020.02.07.20019943. https://doi.org/10.1101/2020.02.07.20019943

27. Chung YS, Barch DM (2016) Frontal-Striatum dysfunction during reward processing: Relationships to amotivation in schizophrenia. J Abnorm Psychol 125:1-17. https://doi.org/10.1037/abn0000137

28. Park IH, Chun JW, Park HJ, et al (2015) Altered cingulo-striatal function underlies reward drive deficits in schizophrenia. Schizophr Res 161:229-236. https://doi.org/10.1016/j.schres.2014.11.005

29. Guessoum SB, Le Strat Y, Dubertret C, Mallet J (2020) A transnosographic approach of negative symptoms pathophysiology in schizophrenia and depressive disorders. Prog. Neuro-

Psychopharmacology Biol. Psychiatry 99:109862 
30. Segarra N, Metastasio A, Ziauddeen H, et al (2016) Abnormal Frontostriatal Activity During Unexpected Reward Receipt in Depression and Schizophrenia: Relationship to Anhedonia. Neuropsychopharmacology 41:2001-2010. https://doi.org/10.1038/npp.2015.370

31. Reckless GE, Andreassen OA, Server A, et al (2015) Negative symptoms in schizophrenia are associated with aberrant striato-cortical connectivity in a rewarded perceptual decision-making task. NeuroImage Clin 8:290-297. https://doi.org/10.1016/j.nicl.2015.04.025

32. Xu P, Klaasen NG, Opmeer EM, et al (2019) Intrinsic mesocorticolimbic connectivity is negatively associated with social amotivation in people with schizophrenia. Schizophr Res 208:353-359. https://doi.org/10.1016/j.schres.2019.01.023

33. Bergé D, Mané A, Salgado P, et al (2016) Predictors of Relapse and Functioning in First-Episode Psychosis: A Two-Year Follow-Up Study. Psychiatr Serv 67:227-233. https://doi.org/10.1176/appi.ps.201400316

34. American Psychiatric Association (2013) Diagnostic and statistical manual of mental disorders (5th ed.). Washington, DC

35. Kirkpatrick B, Strauss GP, Nguyen L, et al (2011) The brief negative symptom scale: psychometric properties. Schizophr Bull 37:300-5. https://doi.org/10.1093/schbul/sbq059

36. Jones SH, Thornicroft G, Coffey M, Dunn G (1995) A Brief Mental Health Outcome Scale: Reliability and Validity of the Global Assessment of Functioning (GAF). Br J Psychiatry 166:654659. https://doi.org/10.1192/bjp.166.5.654

37. First MB, Spitzer RL, Gibbon M, Williams JBW (1997) Structured Clinical Interview for DSM-IV Axis I disorders (SCID I). New York: Biometric Research Department.

38. Sheehan D V, Lecrubier Y, Sheehan KH, et al (1998) The Mini-International Neuropsychiatric Interview (M.I.N.I.): the development and validation of a structured diagnostic psychiatric interview for DSM-IV and ICD-10. J Clin Psychiatry 22-33; quiz 34-57

39. Kay SR, Fiszbein A, Opler LA (1987) The positive and negative syndrome scale (PANSS) for schizophrenia. Schizophr Bull 13:261-76

40. Kleiner M, Brainard D, Pelli D, et al (2007) What's new in psychtoolbox-3. Perception 36:1-16

41. Bergé D, Pretus C, Güell X, et al Reduced willingness to invest effort in schizophrenia with high negative symptoms regardless of reward stimulus presentation and reward value. submitted

42. Kapur S, Seeman P (2001) Does fast dissociation from the dopamine D2 receptor explain the action of atypical antipsychotics?: A new hypothesis. Am. J. Psychiatry 158:360-369

43. Yarkoni T, Poldrack RA, Nichols TE, et al (2011) NeuroSynth: a new platform for large-scale automated synthesis of human functional neuroimaging data. Front Neuroinform 5:. https://doi.org/10.3389/conf.fninf.2011.08.00058

44. McLaren DG, Ries ML, Xu G, Johnson SC (2012) A generalized form of context-dependent psychophysiological interactions (gPPI): A comparison to standard approaches. Neuroimage 61:1277-1286. https://doi.org/10.1016/j.neuroimage.2012.03.068

45. Guitart-Masip M, Fuentemilla L, Bach DR, et al (2011) Action dominates valence in anticipatory 
representations in the human striatum and dopaminergic midbrain. J Neurosci 31:7867-75. https://doi.org/10.1523/JNEUROSCI.6376-10.2011

46. Grahn JA, Parkinson JA, Owen AM (2008) The cognitive functions of the caudate nucleus. Prog Neurobiol 86:141-155. https://doi.org/10.1016/j.pneurobio.2008.09.004

47. Delgado MR, Stenger VA, Fiez JA (2004) Motivation-dependent responses in the human caudate nucleus. Cereb Cortex 14:1022-1030. https://doi.org/10.1093/cercor/bhh062

48. Dosenbach NUF, Visscher KM, Palmer ED, et al (2006) A Core System for the Implementation of Task Sets. Neuron 50:799-812. https://doi.org/10.1016/j.neuron.2006.04.031

49. Sadaghiani S, D’Esposito M (2015) Functional characterization of the cingulo-opercular network in the maintenance of tonic alertness. Cereb Cortex 25:2763-2773. https://doi.org/10.1093/cercor/bhu072

50. Shukla DK, Chiappelli JJ, Sampath H, et al (2019) Aberrant Frontostriatal Connectivity in Negative Symptoms of Schizophrenia. Schizophr Bull 45:1051-1059. https://doi.org/10.1093/schbul/sby165

51. Van Den Heuvel MP, Sporns O, Collin G, et al (2013) Abnormal rich club organization and functional brain dynamics in schizophrenia. JAMA Psychiatry 70:783-792. https://doi.org/10.1001/jamapsychiatry.2013.1328

52. Zhou Y, Zeidman P, Wu S, et al (2018) Altered intrinsic and extrinsic connectivity in schizophrenia. NeuroImage Clin 17:704-716. https://doi.org/10.1016/j.nicl.2017.12.006

53. Van Den Heuvel MP, Fornito A (2014) Brain networks in schizophrenia. Neuropsychol. Rev. 24:3248

54. Fornito A, Zalesky A, Pantelis C, Bullmore ET (2012) Schizophrenia, neuroimaging and connectomics. Neuroimage 62:2296-2314

55. Carpenter WT, Heinrichs DW, Alphs LD (1985) Treatment of Negative Symptoms. Schizophr Bull $11: 440-452$

56. Kirschner M, Aleman A, Kaiser S (2017) Secondary negative symptoms - A review of mechanisms, assessment and treatment. Schizophr. Res. 186:29-38

57. Fervaha G, Takeuchi H, Lee J, et al (2015) Antipsychotics and Amotivation. Neuropsychopharmacology 40:1539-1548. https://doi.org/10.1038/npp.2015.3 


\section{Tables}

Table 1. Demographic and clinical data of the schizophrenia patients (SQZ) sample and control sample.

\begin{tabular}{lcccc}
\hline \multicolumn{1}{c}{ Characteristic } & SQZ & Controls & Statistic & p-value \\
\hline $\mathrm{N}$ & 28 & 20 & & \\
Age $($ years \pm sd) & $37.21(8.62)$ & $31.35(8.59)$ & $\mathrm{t}(46)=-2.33$ & 0.025 \\
Sex $(\%$ of women) & 35.7 & 50 & $\chi^{2}(1)=.980$ & .322 \\
BNSS score (score \pm sd) & $19.57(11.73)$ & - & - & - \\
$\quad$ Expression domain & $7.69(6.69)$ & - & - & - \\
\multicolumn{1}{c}{ Motivation domain } & $11.61(6.47)$ & - & - & - \\
GAF (score \pm sd) & $64.07(14.08)$ & - & - & - \\
$\begin{array}{l}\text { Antipsychotic dose } \\
(\text { CPZ100meq } \pm \text { sd) }\end{array}$ & $2.57(2.45)$ & - & & - \\
\hline
\end{tabular}




\begin{tabular}{|c|c|c|c|c|c|c|}
\hline & \multicolumn{3}{|c|}{ MNI coordinates } & \multirow{2}{*}{$\mathbf{T} \max$} & \multirow{2}{*}{$\begin{array}{l}\text { cluster } \\
\text { size }\end{array}$} & \multirow{2}{*}{$\begin{array}{l}\text { cluster-level } \\
\text { FWE p-value }\end{array}$} \\
\hline & $\mathbf{x}$ & $\mathbf{y}$ & $\mathbf{z}$ & & & \\
\hline \multicolumn{7}{|c|}{ Reward presentation: 1 euro $>1$ cent; control $>$ schizophrenia } \\
\hline L caudate & -18 & 24 & 12 & 5.44 & 309 & $0.060 *$ (n.s.) \\
\hline L insula & -26 & 18 & 20 & 4.98 & & \\
\hline $\mathrm{L} \sup \mathrm{ACC}$ & -16 & 34 & 14 & 3.47 & & \\
\hline R caudate & 18 & 12 & 22 & 4.79 & 1003 & 0.001 \\
\hline $\mathrm{R} \sup \mathrm{ACC}$ & 22 & 30 & 18 & 4.48 & & \\
\hline $\begin{array}{l}\mathrm{R} \text { inferior frontal gryus } \\
\text { (pars triangularis) }\end{array}$ & 30 & 14 & 22 & 4.26 & & \\
\hline \multicolumn{7}{|c|}{ Effort selection: 1 euro > 1 cent; control > schizophrenia } \\
\hline L caudate & -18 & 24 & 12 & 5.00 & 618 & 0.009 \\
\hline L caudate & -24 & -2 & 28 & 4.59 & & \\
\hline L insula & -26 & 18 & 20 & 4.56 & & \\
\hline $\begin{array}{l}\mathrm{R} \text { inferior frontal gryus } \\
\text { (pars triangularis) }\end{array}$ & 30 & 12 & 22 & 4.67 & 1595 & $<0.001$ \\
\hline R caudate & 18 & 8 & 26 & 4.40 & & \\
\hline $\mathrm{R}$ middle frontal gyrus & 24 & 30 & 20 & 4.38 & & \\
\hline
\end{tabular}

Table 2. Neuroimaging results of the reward value contrast during reward presentation and effort selection in schizophrenia patients compared to controls. $\mathrm{L}=$ left, $\mathrm{R}=$ right. 
Table 3. Neuroimaging effective connectivity results of the reward value contrast during effort selection in schizophrenia patients compared to controls. $\mathrm{L}=$ left, $\mathrm{R}=$ right.

\begin{tabular}{|c|c|c|c|c|c|}
\hline & \multicolumn{3}{|c|}{ MNI coordinates } & \multirow{2}{*}{$\begin{array}{l}\text { T(df } \\
=46)\end{array}$} & \multirow{2}{*}{$\begin{array}{c}\text { p-FDR } \\
\text { (seed-level } \\
\text { correction) }\end{array}$} \\
\hline & $\mathbf{x}$ & $\mathbf{y}$ & $\mathbf{z}$ & & \\
\hline \multicolumn{6}{|c|}{ Effort selection: 1euro > 1 cent; control > schizophrenia } \\
\hline \multicolumn{6}{|c|}{$\begin{array}{l}\text { Seed: bilateral caudate (neurosynth "reward" mask) } \\
\mathrm{F}(10)(37)=2.62 ; \text { Intensity }=10.05 ; \text { Size }=4\end{array}$} \\
\hline $\begin{array}{l}\text { Frontoparietal network - R lateral } \\
\text { prefrontal cortex }\end{array}$ & 41 & 38 & 30 & 3.03 & 0.030 \\
\hline $\begin{array}{l}\text { Salience network - R supramarginal } \\
\text { gyrus }\end{array}$ & 62 & -35 & 32 & 2.34 & 0.045 \\
\hline $\begin{array}{l}\text { Frontoparietal network - L posterior } \\
\text { parietal cortex }\end{array}$ & -46 & -58 & 49 & 2.34 & 0.045 \\
\hline $\begin{array}{l}\text { Frontoparietal network }-\mathrm{R} \text { posterior } \\
\text { parietal cortex }\end{array}$ & 52 & -52 & 45 & 2.33 & 0.045 \\
\hline \multicolumn{6}{|c|}{$\begin{array}{l}\text { Seed: Dorsal attention }-\mathbf{L} \text { inferior parietal sulcus } \\
\mathrm{F}(10)(37)=1.53 ; \text { Intensity }=3.05 ; \text { Size }=1\end{array}$} \\
\hline $\begin{array}{l}\text { Frontoparietal network - R lateral } \\
\text { prefrontal cortex }\end{array}$ & 41 & 38 & 30 & 3.05 & 0.029 \\
\hline
\end{tabular}




\section{Figures}

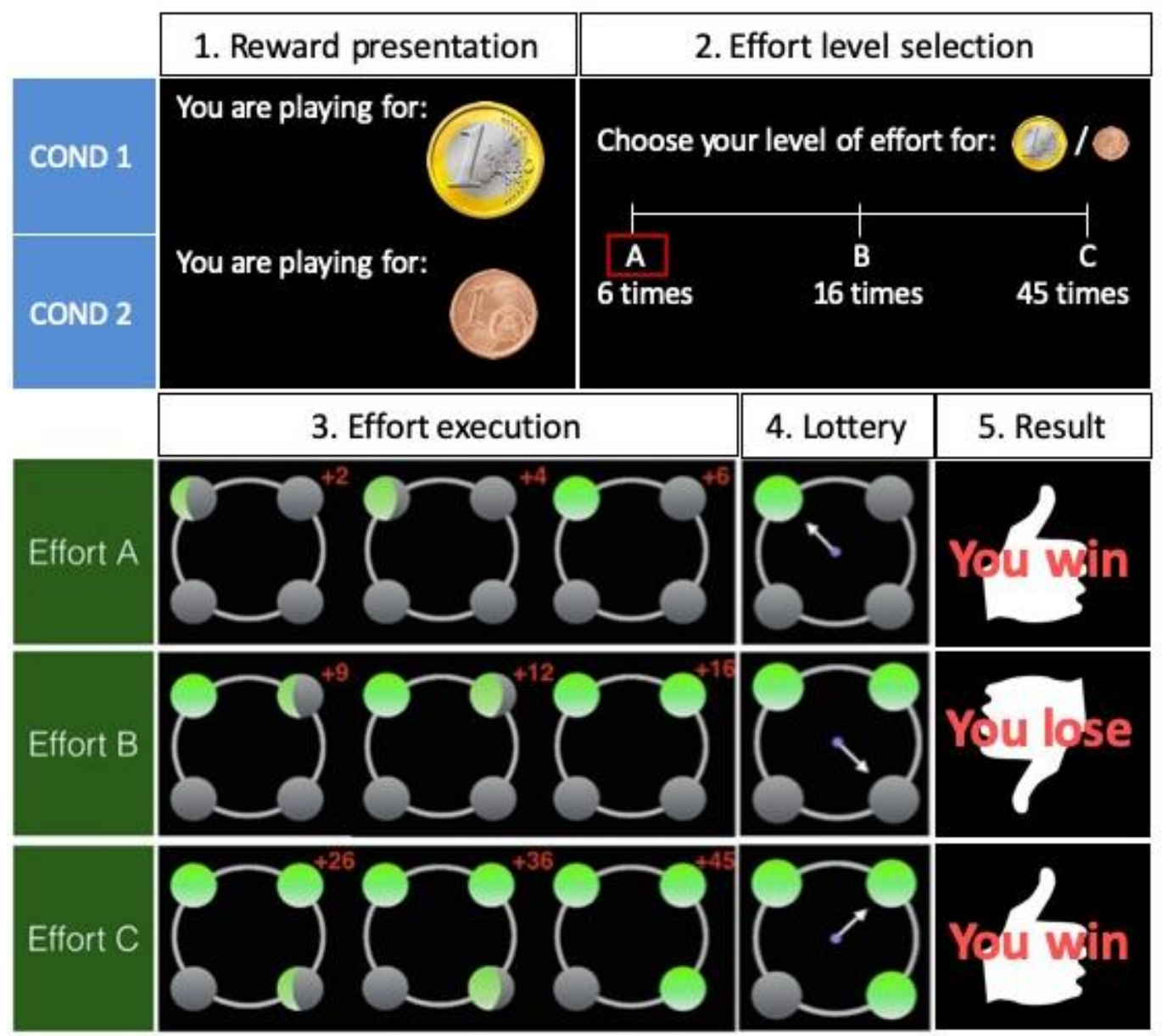

Fig1. Functional magnetic resonance paradigm design, including a lottery game where the participant plays for 1euro in half of the trials and for 1 cent in the other half. The amount of money at stake in a given trial is displayed in the reward presentation screen ("You are playing for: 1 euro/1cent", 750ms). In the "Effort level selection" screen $(2000 \mathrm{~ms})$, the participant is inquired to choose a level of effort (A, B or C) corresponding to the number of times she is willing to press a key in order to increase her odds of winning the game $(6,16$ and 45 , respectively) ("Choose your level of effort for 1euro/1 cent"). A reminder of the condition is displayed in the upper right corner (either 1 euro or 1 cent coin). Next, the participant is required to repeatedly press the key according to her selected effort level causing the circles to turn green ("Effort execution" screen, up to $8000 \mathrm{~ms}$ ). Effort level A requires 1 out of the 4 circles to turn green (6 key presses), Effort B requires 2 out of 4 (16 key presses) and Effort $C$ requires 3 out of 4 (45 key presses). Subsequent circles are increasingly harder to turn green: the first circle "costs" 6 key presses, the second circle 10 key presses [16 left key presses for Effort B - 6 completed key presses for Effort A] and the third circle 29 key presses [45 left key presses for Effort C - 16 completed key presses for Effort A and B]. A lottery game follows (900-1200ms), where the participant wins if the spike hits at a green circle. At the end of the trial, the result ("You win"/"You lose", $750 \mathrm{~ms}$ ) and the participant's accumulated gain (not shown in this figure, $750 \mathrm{~ms}$ ) are displayed. 


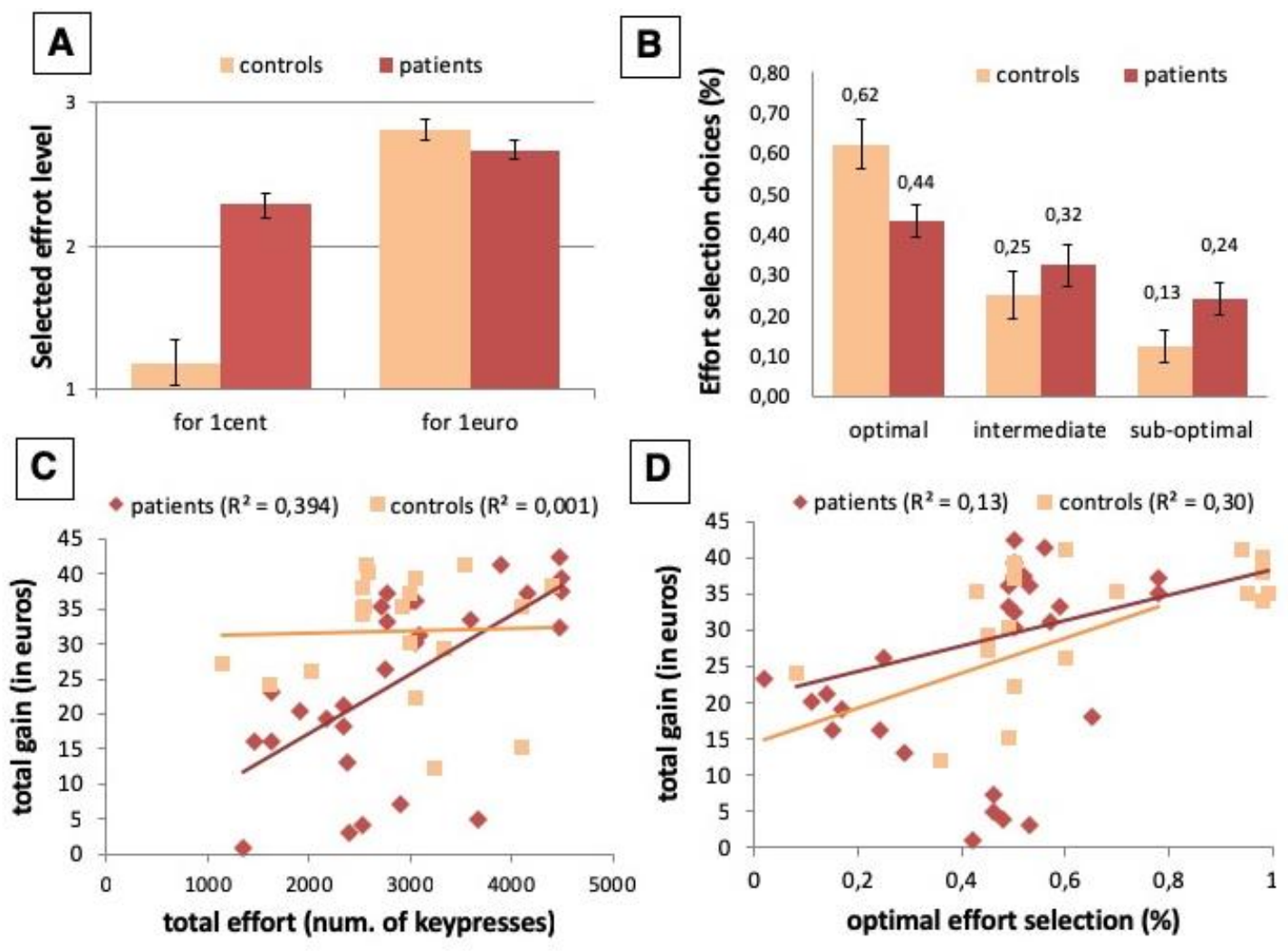

Fig2. Behavioral data analysis results, including (A) lower effort levels for 1 euro and higher effort levels for 1 cent in patients compared to controls (Wilk's Lambda $=0.746, F(44,1)=15, p<0.001$ ), (B) lower percentage of optimal choices (maximal effort for leuro and minimal effort for 1 cent) in patients compared to controls ( $\mathrm{t}(46)=2.882, \mathrm{p}=0.006)$, and no between-group differences in intermediate or suboptimal choices, (C) positive correlation between total gain (in euros) and total effort in patients but not in controls, and (D) positive correlation between total gain (in euros) and optimal choices in controls but not in patients. 

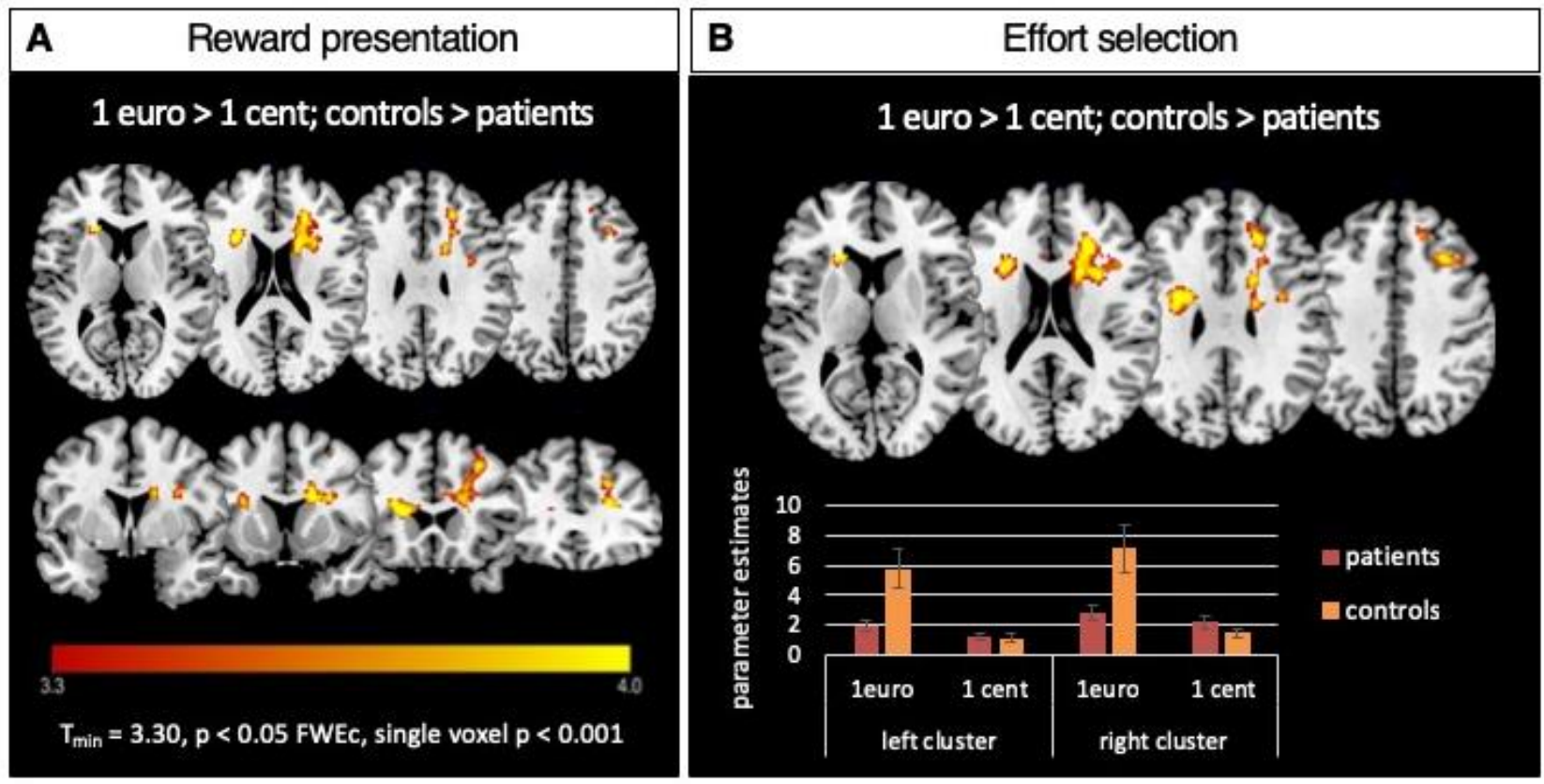

Fig3. Neuroimaging results: (A) Reward presentation events yielded significantly higher activity in the bilateral caudate as well as part of the insula, the anterior cingulate cortex and the inferior prefrontal cortex in the 1euro (vs. 1cent) condition in controls compared to patients $\left(\mathrm{T}_{\min }=3.30, \mathrm{p}<0.05\right.$ FWEc, single voxel $\mathrm{p}<$ 0.001). (B) Effort selection events revealed a similar pattern of between-group neural activity differences, with parameter estimates of both left and right clusters showing increased neural recruitment in response to 1 euro versus 1 cent events in controls, a discrepancy that was not observed in schizophrenia patients. 

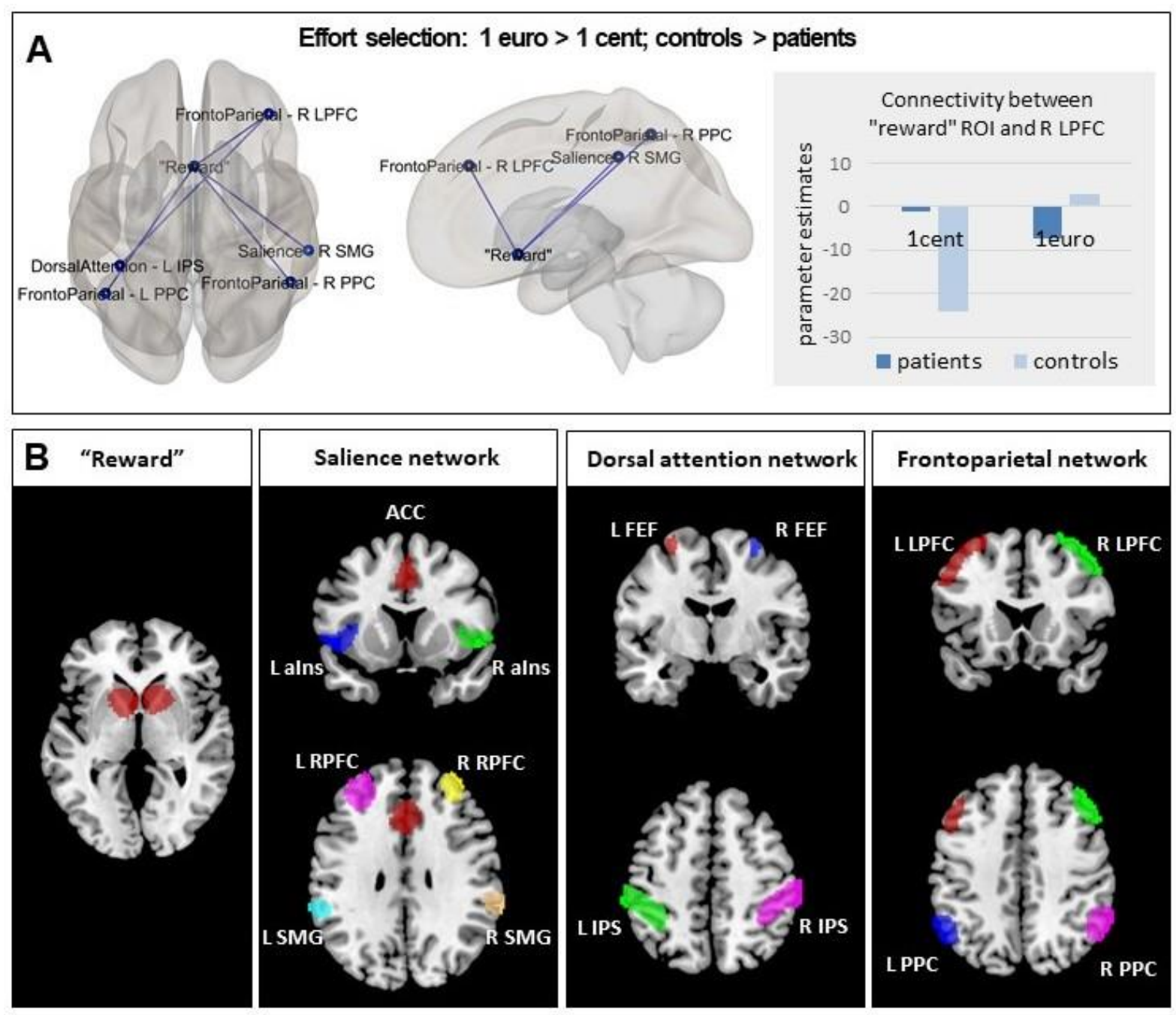

Fig4. Effective connectivity results: (A) The gPPI analysis revealed decreased connectivity between the bilateral caudate and both frontoparietal network regions (right lateral prefrontal cortex and bilateral posterior parietal cortex) and salience network regions (right supramarginal gyrus), as well as decreased connectivity between nodes in the dorsal attention network (left inferior parietal sulcus) and in the frontoparietal network (right lateral prefrontal cortex) in patients compared to controls during effort selection in the 1 euro compared to the 1 cent condition ( $\mathrm{p}<0.05$ FDR seed-level corrected), (B) Sixteen ROIs were included in the gPPI analysis, encompassing a functional mask for "reward" extracted from Neurosynth.org, seven ROIs in the salience network, four ROIs in the dorsal attention network and four ROIs in the frontoparietal network. Different colors have been used to visually distinguish ROIs within each network with no interrelation across different networks. Bilat Caud $=$ bilateral Caudate, $\mathrm{ACC}=$ anterior cingulate cortex, aIns $=$ anterior insula, $\mathrm{RPFC}=$ rostral prefrontal cortex, $\mathrm{SMG}=$ supramarginal gyrus, $\mathrm{FEF}=$ frontal eye fields, IPS $=$ inferior parietal sulcus, $\mathrm{LPFC}=$ lateral prefrontal cortex, $\mathrm{PPC}=$ posterior parietal cortex $\mathrm{L}=$ left, $\mathrm{R}=$ right. 